\section{Effect of laser suture lysis on filtration openings: a prospective three- dimensional anterior segment optical coherence tomography study}

H-k Cho',2, S Kojima', T Inoue ${ }^{1}$, A Fukushima1, C Kee $^{2}$ and H Tanihara ${ }^{1}$
${ }^{1}$ Department of Ophthalmology, Faculty of Life Sciences, Kumamoto University, Kumamoto, Japan

${ }^{2}$ Department of Ophthalmology, Samsung Medical Center, Sungkyunkwan University School of Medicine, Seoul, Republic of Korea

Correspondence:

$T$ Inoue, Department of Ophthalmology, Faculty of Life Sciences, Kumamoto University, 1-1-1, Honjo, Chuo-ku, Kumamoto 860-8556, Japan Tel: +81 96373 5247; Fax: +81963735249. E-mail: noel@da2.so-net.ne.jp

Received: 4 January 2015 Accepted in revised form: 10 June 2015 Published online: 24 July 2015

\begin{abstract}
Purpose To investigate the effects of laser suture lysis (LSL) on filtration openings after trabeculectomy.

Methods Prospective study analyzing the changes in the location and width of filtration openings, fluid cavity height, total bleb height, bleb wall thickness, and bleb wall intensity before and after LSL using threedimensional anterior segment optical coherence tomography (3D AS-OCT). Results Fourteen patients had clear scleral flap image analysis. As five patients underwent LSL twice and two patients underwent LSL thrice, 23 comparison studies were possible. After LSL the intraocular pressure (IOP) decreased $(P=0.0015)$ from $20.5 \pm 5.3$ to $14.9 \pm 6.4 \mathrm{~mm} \mathrm{Hg}$, and the fluid cavity height increased significantly from $0.2 \pm 0.2 \mathrm{~mm}$ to $0.3 \pm 0.1 \mathrm{~mm}(P=0.0094)$. Other bleb parameters were not significantly different when comparing before and after LSL. When the IOP reduction ratio was $>25 \%$ following LSL, the width of the filtration openings on the LSL side, the total bleb height, and the fluid cavity height increased $(P=0.0273,0.0342$, and 0.0024 , respectively). In multiple regression analysis the changes in fluid cavity height, the wall thickness, the wall intensity, and the width of the filtration opening were positively associated with the IOP reduction rate $(P=0.0428,0.0226,0.0420$, and 0.0356 , respectively). Conclusions 3D AS-OCT allowed a detailed examination of the internal morphology of filtration blebs and openings before and after LSL. The changes in the internal morphology
\end{abstract}

were closely associated with the success of LSL to decrease IOP.

Eye (2015) 29, 1220-1225; doi:10.1038/eye.2015.129; published online 24 July 2015

\section{Introduction}

Trabeculectomy is a procedure of choice in the surgical management of glaucoma refractory to medical and laser treatments. ${ }^{1}$ Good intraocular pressure (IOP) control after filtration surgery is generally associated with the existence of a filtration bleb and its morphology. ${ }^{2,3}$

Recognizing the importance of bleb appearance in relation to the surgical outcome, several classifications of bleb morphology based on slit-lamp grading have been proposed. 4,5 The visualization of internal bleb structures was first reported using ultrasound biomicroscopy (UBM). ${ }^{6}$ However, UBM is limited by the need for contact, especially in the early postoperative period, and the axial resolution does not exceed $25 \mu \mathrm{m}$.

The recent development of anterior segment optical coherence tomography (AS-OCT) has enabled non-contact, high-resolution imaging of anterior segment structures. ${ }^{7,8}$ However, the previous studies used two-dimensional (2D) OCT, so the filtration openings could not be identified because of the limited number of sections (a radial section perpendicular to the limbus and/or a section tangential to the limbus through the site of maximal bleb elevation). ${ }^{7,8}$ With three-dimensional (3D) AS-OCT, aqueous outflow openings at the margin of scleral flaps can be visualized more clearly using en face OCT images. ${ }^{9,10}$ In our previous studies we obtained 
512 3D scans that were rotated using newly developed software (CASIA bleb-assessment software, Tomey) and matched with reconstructed C-scan images (tomographic image of the slice plane perpendicular to the depth direction) to precisely identify the filtration openings on the scleral flap. ${ }^{11-14}$ Filtration openings were identified in $95 \%$ (118 of 124) of the eyes; the location of the openings was also visualized. We found that the pattern of filtration openings was not affected by the residual suture after laser application in this small case series of laser suture lysis (LSL). In comparison, studies from Singapore reported an increased bleb height after LSL using 2D AS-OCT images. ${ }^{15,16}$

Therefore, in this prospective study, we investigated the effect of LSL on the scleral flap using 3D AS-OCT and the CASIA software. The changes in the location and width of the filtration opening were inspected, along with the bleb height and bleb wall intensity before and after LSL. These findings might assist in determining a strategy for LSL as postoperative treatment of trabeculectomy.

\section{Materials and methods}

\section{Patients}

This prospective study was approved by the Institutional Review Board of Kumamoto University, Japan, and was registered with the University Hospital Medical Information Network Clinical Trials Registry of Japan (ID UMIN000006008; date of access and registration, 21 July 2011). The protocol adhered to the tenets of the Declaration of Helsinki Principles, and written informed consent was obtained from all patients. Patients who underwent LSL after a trabeculectomy were enrolled in this study. If both eyes in the same patient met the inclusion criteria, the eye that first underwent trabeculectomy was included in the study.

\section{Surgical procedures}

For the trabeculectomy, a fornix-based conjunctival flap was first created and a 4-mm wide half-layer triangular scleral flap was created. Mitomycin C $(0.4 \mathrm{mg} / \mathrm{ml})$ was applied on and under the scleral flap and under the conjunctiva for $4 \mathrm{~min}$; this was followed by irrigation with $200 \mathrm{ml}$ physiological saline. A deep limbal block was excised to create a fistula in the anterior chamber; then, a peripheral iridectomy was performed. The scleral and conjunctival flaps were sutured with 10-0 Nylon. Three sutures were placed on the triangular scleral flap, one on top of the flap and one in the middle of each side of the flap. Patients underwent LSL when the postoperative IOP exceeded $15 \mathrm{~mm} \mathrm{Hg}$ or the bleb was poorly formed. LSL was performed with an argon laser (Lumenis, Yokneam, Israel) and a Blumenthal lens (Volk Optical, Mentor, OH, USA) by each observer who was blinded to the 3D AS-OCT results. The observer independently decided which suture to cut.

\section{Data collection}

We conducted slit-lamp examinations and measured IOP daily until 14 days after the trabeculectomy. 3D AS-OCT images of the blebs were obtained immediately prior to each LSL procedure and 14 days after the trabeculectomy.

The internal bleb structures were assessed using 3D AS-OCT (CASIA; Tomey, Nagoya, Japan), as reported. ${ }^{11}$ Briefly, the area examined included the entire scleral flap and bleb. Three reviewers evaluated the complete 3D images of the internal structure of the filtration blebs, and independently assessed filtration openings. When opinions about filtration openings differed among the reviewers, a majority decision was used. Filtration openings were identified by the presence of pits or troughs in fluid-filled cavities in both horizontal and vertical rasters and by a corresponding C-scan image of the scleral flap margins in the bleb using the installed CASIA bleb-assessment software (ver. $4.0 \mathrm{~L}$; Tomey), which allowed us to approve arbitrary cross-section images from the restructured 3D data. Consequently, we could identify filtration openings on the plane along the scleral flap (Figure 1). Those blebs in which filtration openings could be identified by 3D AS-OCT were termed type F; those blebs in which filtration openings could not be identified were termed as described in our previous study. ${ }^{11}$ Briefly, non-functioning flat-shaped blebs without fluid-filled cavity, blebs had an elevated bleb wall that was sometimes of high reflectivity, and blebs of which filtration openings were masked by a sponge-like structure exhibiting low reflectivity were defined as type $\mathrm{N}, \mathrm{H}$, and S, respectively. In the type F blebs, the width of the filtration openings on the scleral flap and the distance between the top of the scleral flap and the center of the opening was defined as the top filtration distance (TFD; Figure 1). Total bleb height, fluid cavity height, bleb wall thickness, and bleb wall intensity were measured in the horizontal image at the center of the filtration opening. In type $\mathrm{N}$ blebs, the width of the filtration opening was defined as 0 . The average value of each bleb parameter among the three reviewers was used in further analyses. Data obtained immediately prior to LSL were compared with data immediately before a subsequent LSL, or were compared with data obtained 14 days after trabeculectomy if the LSL was the final procedure performed during the period. 
a

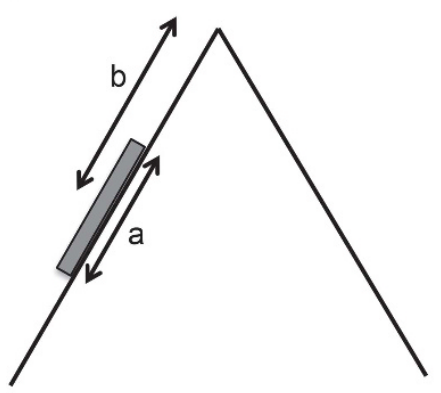

a. Filtration opening width

b. TFD

Box. Filtration opening b

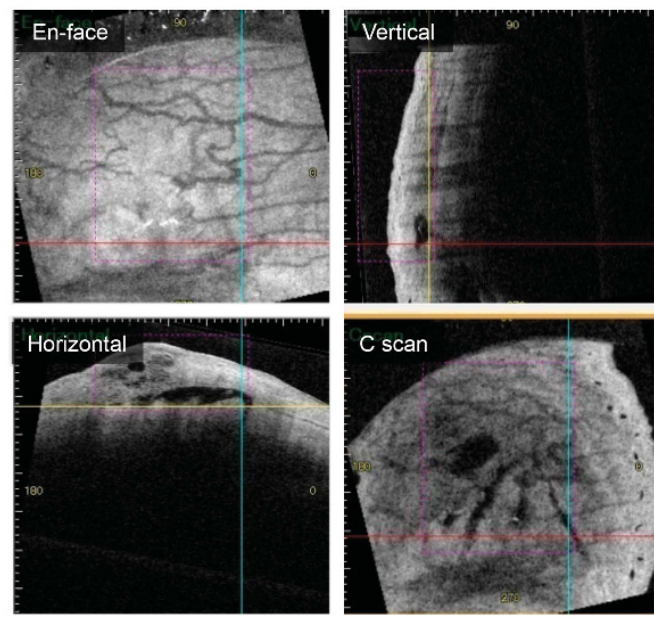

Figure 1 (a) Diagram showing the filtration opening on the scleral flap and the definition of the top filtration distance (TFD); i.e., the center of the filtration opening on the scleral flap. (b) OCT images of the bleb with filtration opening on the scleral flap. Red, blue, and yellow lines indicate the horizontal, vertical, and C-scan axes of OCT, respectively. Filtration openings were identified by the presence of pits or troughs in fluid-filled cavities in both horizontal and vertical rasters and by a corresponding C-scan image of the scleral flap margins in the bleb using the installed software, which allowed us to approve arbitrary cross-section images from the restructured 3D data. Consequently, we could identify filtration openings on the plane along the scleral flap (crossing points of the color lines).

\section{Statistical analysis}

The patients' characteristics were compared using the Wilcoxon signed-rank test. Factors associated with the percentage reduction in the IOP between before and after LSL were assessed by multiple regression analysis. Values of $P<0.05$ were considered to indicate statistical significance.

\section{Results}

Sixteen eyes of 16 patients who underwent LSL after trabeculectomy were included (Supplementary Table 1). From these, one patient had type $\mathrm{H}$ blebs, and another patient moved their eye during the examination and were excluded from the analysis. Ultimately, 14 patients had type F or N blebs both before and after LSL in AS-OCT images. Seven patients were included in our past prospective study. ${ }^{13}$ Six patients had a history of cataract surgery prior to the subjective trabeculectomy. Of those, two eyes received vitrectomy at that time. No eye received glaucoma surgery before the subjective trabeculectomy. Seven patients underwent LSL at least twice, allowing 23 comparisons to be made before and after LSL. The mean $( \pm$ SD) age was $68.3 \pm 10.7$ years. The glaucoma was diagnosed as four primary open-angle glaucoma, five exfoliation glaucoma, one primary angle closure glaucoma, and secondary glaucoma (two uveitic glaucoma and two neovascular glaucoma due to proliferative diabetic retinopathy) in four eyes each.
The IOP decreased significantly after LSL from $20.5 \pm 5.3$ to $14.9 \pm 6.4 \mathrm{~mm} \mathrm{Hg}(P=0.0015)$.

The TFD was $1.7 \pm 0.5 \mathrm{~mm}$ before LSL and $1.8 \pm 0.5 \mathrm{~mm}$ after LSL $(P=0.1780)$. The widths of the filtration openings before and after LSL were $1.0 \pm 1.5$ and $1.2 \pm 1.3 \mathrm{~mm}$, respectively $(P=0.2573)$. On the applied side, the width of the filtration openings was $0.5 \pm 0.9 \mathrm{~mm}$ before LSL and $0.9 \pm 0.8 \mathrm{~mm}$ after LSL $(P=0.0554)$.

The corresponding values for the opposite side were $0.5 \pm 0.8$ and $0.4 \pm 0.6 \mathrm{~mm}$, respectively $(P=0.4316)$. There were no significant changes in the width of the filtration openings before and after LSL on both the LSL and opposite sides. The total bleb height was $0.6 \pm 0.3 \mathrm{~mm}$ before LSL and $0.7 \pm 0.4 \mathrm{~mm}$ after LSL $(P=0.1014)$. The fluid cavity height before and after LSL was $0.2 \pm 0.2$ and $0.3 \pm 0.1 \mathrm{~mm}$, respectively $(P=0.0094)$. Bleb wall thickness was $0.4 \pm 0.2 \mathrm{~mm}$ before LSL and $0.4 \pm 0.3 \mathrm{~mm}$ after LSL $(P=0.7348)$. The bleb wall intensity (optical density) was $157.9 \pm 24.4$ before LSL and $157.9 \pm 42.2$ after LSL $(P=0.5749)$. Overall, the fluid cavity height increased significantly after LSL, while the other parameters did not differ significantly between before and after LSL.

As a post hoc analysis, we subdivided the procedures into two groups based on the median of IOP reduction ratio: (1) IOP decreased $>25 \%$ after LSL (successful group, $n=12$, from 27.3 to $85.0 \%$ ) and (2) IOP decreased $\leq 25 \%$ (unsuccessful group, $n=11$, from -29.4 to $5.9 \%$ ). There were no significant differences in background factors and bleb parameters before LSL between the groups. In the successful group, the IOP decreased after LSL from $21.8 \pm 5.8$ to $10.3 \pm 4.2 \mathrm{~mm} \mathrm{Hg}(P=0.0005)$. 
The TFD was $1.7 \pm 0.6 \mathrm{~mm}$ before LSL and $1.8 \pm 0.5 \mathrm{~mm}$ after LSL $(P=0.7910)$. The total widths of the filtration openings before and after LSL were $1.1 \pm 1.3$ and $1.7 \pm 1.2 \mathrm{~mm}$, respectively $(P=0.0664)$. The filtration opening width on the LSL side increased significantly after LSL from $0.6 \pm 0.8$ to $1.3 \pm 0.6 \mathrm{~mm}(P=0.0273)$. Moreover, the total bleb height and the fluid cavity height increased significantly after LSL from $0.6 \pm 0.2$ and $0.2 \pm 0.2 \mathrm{~mm}$ to $0.8 \pm 0.4$ and $0.3 \pm 0.2 \mathrm{~mm}(P=0.0342$ and 0.0024 , respectively). The results were not materially different for other IOP cutoffs, 20 and 30\% reduction, except in total bleb height, which was not significantly different with $30 \%$ of cutoff. Representative 3D AS-OCT images before and after successful LSL are shown in Figure 2. In the unsuccessful group, the bleb parameters did not change significantly before and after LSL

(Table 1).

To identify the associations of the variation of the bleb parameters before and after LSL with the reduction rate in IOP, we conducted multiple regression analysis. The changes in fluid cavity height, the wall thickness, the wall intensity, and the width of the filtration opening were positively associated with the IOP reduction rate $(P=0.0428,0.0226,0.0420$, and 0.0356 , respectively; Table 2 ).

\section{Discussion}

This study found that the location (TFD) or total width of the filtration opening did not change after LSL. The width of the filtration opening on both the LSL and opposite sides did not change after LSL. These results are consistent with our previous finding that the opening locations were similar despite the pattern of remaining sutures and cut nylon sutures. ${ }^{11}$ Only fluid cavity height increased significantly after LSL. However, from analyzing only the successful procedures (IOP reduction $>25 \%$ ), the width of the filtration openings on the
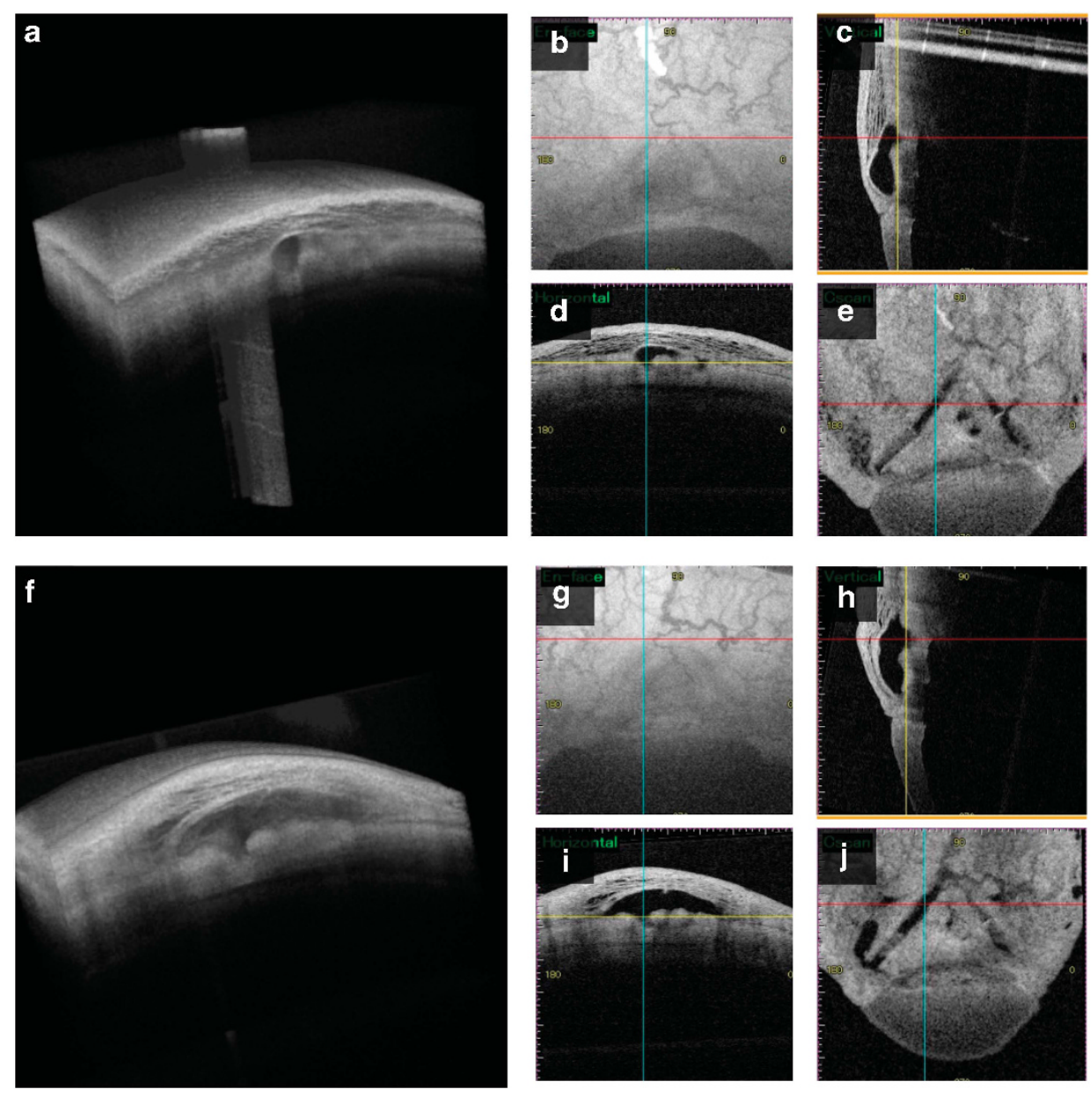

Figure 2 Representative bleb images assessed by three-dimensional anterior segment optical coherence tomography before (a-e) and after (f-j) successful laser suture lysis (LSL). (a and f) 3D images; (b and g) en face images; (c and h) vertical images; (d and i) horizontal images; (e and j) C-scan images. The width of filtration openings, total bleb height, and fluid cavity height were increased after successful LSL. 
Table 1 Changes in bleb parameters before and after LSL in the groups categorized by IOP reduction ratio

\begin{tabular}{|c|c|c|c|c|c|c|}
\hline \multirow{2}{*}{ Parameters } & \multicolumn{3}{|c|}{ Successful group $(\mathrm{n}=12)$} & \multicolumn{3}{|c|}{ Unsuccessful group $(\mathrm{n}=11)$} \\
\hline & Before LSL & After LSL & P-value & Before LSL & After LSL & P-value \\
\hline IOP (mm Hg) & $21.8 \pm 5.8$ & $10.3 \pm 4.2$ & $0.0005^{* *}$ & $19.0 \pm 4.5$ & $19.9 \pm 4.3$ & 0.3828 \\
\hline TFD (mm) & $1.7 \pm 0.6$ & $1.8 \pm 0.5$ & 0.791 & $1.8 \pm 0.3$ & $1.9 \pm 0.6$ & 0.0781 \\
\hline Opening width (mm) & $1.1 \pm 1.3$ & $1.7 \pm 1.2$ & 0.0664 & $0.9 \pm 1.8$ & $0.6 \pm 1.2$ & 0.5625 \\
\hline Opening width on LSL side & $0.6 \pm 0.8$ & $1.3 \pm 0.6$ & $0.0273^{*}$ & $0.4 \pm 1.0$ & $0.5 \pm 0.8$ & 0.8125 \\
\hline Opening width on side opposite LSL & $0.4 \pm 0.7$ & $0.5 \pm 0.7$ & 0.6875 & $0.5 \pm 0.9$ & $0.2 \pm 0.5$ & 0.125 \\
\hline Total bleb height $(\mathrm{mm})$ & $0.6 \pm 0.2$ & $0.8 \pm 0.4$ & $0.0342 *$ & $0.6 \pm 0.3$ & $0.6 \pm 0.3$ & 0.8311 \\
\hline Fluid cavity height (mm) & $0.2 \pm 0.2$ & $0.3 \pm 0.2$ & $0.0024^{*}$ & $0.2 \pm 0.2$ & $0.2 \pm 0.1$ & 0.5566 \\
\hline Bleb wall thickness (mm) & $0.4 \pm 0.1$ & $0.4 \pm 0.2$ & 0.6772 & $0.4 \pm 0.3$ & $0.4 \pm 0.3$ & 0.9854 \\
\hline Bleb wall intensity (optical density) & $157.6 \pm 25.0$ & $152.9 \pm 53.3$ & 0.6772 & $158.2 \pm 25.0$ & $163.3 \pm 26.9$ & 0.9658 \\
\hline
\end{tabular}

Abbreviations: IOP, intraocular pressure; LSL, laser suture lysis; TFD, top filtration distance.

${ }^{* *} P<0.01,{ }^{*} P<0.05$ by Wilcoxon signed-rank test.

Data are shown as means \pm SD.

Table 2 Relations between changes in bleb parameters and the reduction rate of the IOP following LSL calculated by multiple regression analysis

\begin{tabular}{lcl}
\hline Bleb parameter & T-value & P-value \\
\hline$\Delta$ Total bleb height $(\mathrm{mm})$ & -1.59 & 0.131 \\
$\Delta$ Fluid cavity height $(\mathrm{mm})$ & 2.2 & $0.0428^{*}$ \\
$\Delta$ Wall thickness $(\mathrm{mm})$ & 2.52 & $0.0226^{*}$ \\
$\Delta$ Wall intensity (optical density) & 2.21 & $0.0420^{*}$ \\
$\Delta$ Width of filtration opening $(\mathrm{mm})$ & 2.29 & $0.0356^{*}$ \\
$\Delta$ TFD average $(\mathrm{mm})$ & -1.5 & 0.1533 \\
\hline
\end{tabular}

Abbreviations: IOP, intraocular pressure; LSL, laser suture lysis; TFD, top filtration distance.

${ }^{*} P<0.05$.

LSL side, the total bleb height, and the fluid cavity height were significantly increased after LSL. Based on these findings, we postulate that more aqueous humor passes through the filtration opening after successful LSL.

In multiple regression analysis, changes in fluid cavity height, bleb wall thickness, bleb wall intensity, and width of filtration openings were associated with successful LSL, as defined by the IOP reduction ratio. Thus, the LSL procedure was suggested to induce internal changes of the filtering bleb. Especially, it was indicated that the width of filtration openings related to the success of LSL even in the multivariate analysis, and therefore the value was assumed to be one of the key factors for IOP reduction using the LSL procedure.

Singh et al ${ }^{15}$ reported that the total bleb height and bleb wall thickness increased in six out of seven eyes after LSL using 2D AS-OCT, which is partly in discord with our results. One reason for this discrepancy was that we used 3D AS-OCT and the scan used to measure bleb morphology was positioned in the middle of the filtration opening. In addition, they compared the images before LSL and 2 weeks after LSL, while the average interval of the comparison in our study was $5.1 \pm 3.8$ days. As bleb morphology changes with time, ${ }^{13}$ the difference in observation times might explain the contradictory results. Furthermore, Singh et al evaluated the increase in bleb height in a qualitative manner by comparing it with scleral thickness without statistical analysis, while our comparison was quantitative. Collectively, these methodological differences might have led to inconsistent results between the studies.

One of the limitations of our study is the small number of subjects; 23 comparisons were conducted with 14 eyes. Singh et $a l^{15}$ evaluated 7 eyes, whereas Sng et al ${ }^{16}$ investigated 25 eyes. Some of the changes in bleb parameters after LSL might have been insignificant because of the small number of samples. In addition, if the 3D AS-OCT imaging could predict which suture to cut to reduce IOP effectively, it would provide clinical applicability. However, we found no differences in bleb parameters before LSL between successful group and unsuccessful group, possibly because of small sample number. Another limitation is the deviation in the periods between LSL procedure and AS-OCT imaging among cases, which might affect the results in the present study. Finally, the effects of the history of ocular surgeries on the bleb morphology were not analyzed because of small sample size. Collectively, a large, multicenter, randomized clinical trial is required to draw more definite conclusions.

In conclusion, 3D AS-OCT allowed a detailed examination of the internal morphology of filtration blebs and openings before and after LSL, and it was indicated that the changes in the internal morphology were closely associated with successful LSL decreasing IOP. 


\section{Summary}

\section{What was known before}

- To our knowledge, no previous reported study has investigated the changes in the width of filtration openings on the scleral flap following laser suture lysis.

What this study adds

- We showed that the width of the filtration opening was increased after successful laser suture lysis.

\section{Conflict of interest}

The authors declare no conflict of interest.

\section{Author contributions}

$\mathrm{H}-\mathrm{kC}, \mathrm{TI}$, and HT conducted the study design; H-kC, TI, and HT performed the study; H-kC, TI, AF, and SK collected the data, management, analysis, and interpretation; and $\mathrm{H}-\mathrm{kC}, \mathrm{TI}, \mathrm{CK}$, and $\mathrm{HT}$ were involved in the preparation, review, and approval of the manuscript.

\section{Acknowledgements}

This study was approved by the Institutional Review Board of Kumamoto University (Kumamoto, Japan), and was registered with the University Hospital Medical Information Network Clinical Trials Registry of Japan (ID UMIN000006008; date of access and registration, 21 July 2011).

\section{References}

1 Cairns JE. Trabeculectomy. Preliminary report of a new method. Am J Ophthalmol 1968; 66: 673-679.

2 Picht G, Grehn F. Classification of filtering blebs in trabeculectomy: biomicroscopy and functionality. Curr Opin Ophthalmol 1998; 9: 2-8.

3 Vesti E. Filtering blebs: follow up of trabeculectomy. Ophthalmic Surg 1993; 24: 249-255.

4 Cantor LB, Mantravadi A, WuDunn D, Swamynathan K, Cortes A. Morphologic classification of filtering blebs after glaucoma filtration surgery: the Indiana Bleb Appearance Grading Scale. J Glaucoma 2003; 12: 266-271.

5 Wells AP, Crowston JG, Marks J, Kirwan JF, Smith G, Clarke JC et al. A pilot study of a system for grading of drainage blebs after glaucoma surgery. J Glaucoma 2004; 13: 454-460.

6 Pavlin CJ, Harasiewicz K, Foster FS. Ultrasound biomicroscopy of anterior segment structures in normal and glaucomatous eyes. Am J Ophthalmol 1992; 113: 381-389.

7 Leung CK, Yick DW, Kwong YY, Li FC, Leung DY, Mohamed $\mathrm{S}$ et al. Analysis of bleb morphology after trabeculectomy with Visante anterior segment optical coherence tomography. Br J Ophthalmol 2007; 91: 340-344.

8 Singh M, Chew PT, Friedman DS, Nolan WP, See JL, Smith SD et al. Imaging of trabeculectomy blebs using anterior segment optical coherence tomography. Ophthalmology 2007; 114: 47-53.

9 Miura M, Kawana K, Iwasaki T, Kiuchi T, Oshika T, Mori H et al. Three-dimensional anterior segment optical coherence tomography of filtering blebs after trabeculectomy. J Glaucoma 2008; 17: 193-196.

10 Kawana K, Kiuchi T, Yasuno Y, Oshika T. Evaluation of trabeculectomy blebs using 3-dimensional cornea and anterior segment optical coherence tomography. Ophthalmology 2009; 116: 848-855.

11 Inoue T, Matsumura R, Kuroda U, Nakashima K, Kawaji T, Tanihara H. Precise identification of filtration openings on the scleral flap by three-dimensional anterior segment optical coherence tomography. Invest Ophthalmol Vis Sci 2012; 53: 8288-8294.

12 Kojima S, Inoue T, Kawaji T, Tanihara H. Filtration bleb revision guided by 3-dimensional anterior segment optical coherence tomography. J Glaucoma 2014; 23: 312-315.

13 Kojima S, Inoue T, Nakashima K, Fukushima A, Tanihara H. Prospective investigation of filtering blebs using threedimensional anterior-segment optical coherence tomography. JAMA Ophthalmol 2015; 133: 148-156.

14 Nakashima K, Inoue T, Fukushima A, Hirakawa S, Kojima S, Tanihara H. Evaluation of filtering blebs exhibiting transconjunctival oozing using anterior segment optical coherence tomography. Graefes Arch Clin Exp Ophthalmol 2015; 253: 439-445.

15 Singh M, Aung T, Friedman DS, Zheng C, Foster PJ, Nolan WP et al. Anterior segment optical coherence tomography imaging of trabeculectomy blebs before and after laser suture lysis. Am J Ophthalmol 2007; 143: 873-875.

16 Sng CC, Singh M, Chew PT, Ngo CS, Zheng C, Tun TA et al. Quantitative assessment of changes in trabeculectomy blebs after laser suture lysis using anterior segment coherence tomography. J Glaucoma 2012; 21: 313-317. 